\title{
High Throughput Screening of Co-Expressed Gene Pairs with Controlled False Discovery Rate (FDR) and Minimum Acceptable Strength (MAS)
}

\author{
DONGXIAO ZHU, ${ }^{1}$ ALFRED O HERO, ${ }^{2}$ ZHAOHUI S QIN,${ }^{3}$ and ANAND SWAROOP ${ }^{4}$
}

\begin{abstract}
Many exploratory microarray data analysis tools such as gene clustering and relevance networks rely on detecting pairwise gene co-expression. Traditional screening of pairwise co-expression either controls biological significance or statistical significance, but not both. The former approach does not provide stochastic error control, and the later approach screens many co-expressions with excessively low correlation. We have designed and implemented a statistically sound two-stage co-expression detection algorithm that controls both statistical significance (false discovery rate, FDR) and biological significance (minimum acceptable strength, MAS) of the discovered co-expressions. Based on estimation of pairwise gene correlation, the algorithm provides an initial co-expression discovery that controls only FDR, which is then followed by a second stage co-expression discovery which controls both FDR and MAS. It also computes and thresholds the set of FDR $p$-values for each correlation that satisfied the MAS criterion. Using simulated data, we validated asymptotic null distributions of the Pearson and Kendall correlation coefficients and the two-stage errorcontrol procedure; we also compared our two-stage test procedure with another two-stage test procedure using the receiver operating characteristic (ROC) curve. We then used yeast galactose metabolism data to illustrate the advantage of our method for clustering genes and constructing a relevance network. The method has been implemented in an $R$ package "GeneNT" that is freely available from the Comprehensive R Archive Network (CRAN): www.cran.r-project.org/.
\end{abstract}

Key words: false discovery rate confidence interval, relevance network, error control, gene pathway.

\section{INTRODUCTION}

$\mathbf{T}$ The emergence AND DEVELOPMENT OF DNA microarray technology (Affymetrix oligonucleotide expression arrays and cDNA arrays) enable researchers to interrogate gene expression levels

\footnotetext{
${ }^{1}$ Bioinformatics Program, University of Michigan, Ann Arbor, MI 48109.

${ }^{2}$ Departments of EECS and Statistics, University of Michigan, Ann Arbor, MI 48109.

${ }^{3}$ Center for Statistical Genetics and Department of Biostatistics, University of Michigan, Ann Arbor, MI 48109.

${ }^{4}$ Departments of Ophthalmology, Visual Sciences and Human Genetics, University of Michigan, Ann Arbor, MI 48109 .
} 
simultaneously on the genome scale (Lockhart et al., 1996; Schena et al., 1995; DeRisi et al., 1997). The development of statistically sound and biologically meaningful techniques to analyze gene expression data is essential for transforming raw experimental data into scientific knowledge. Gene expression data have been subjected to a variety of statistical analyses, such as detecting differentially expressed genes (e.g., Tusher et al. [2001], Zareparsi et al. [2004]), clustering genes/samples (e.g., Eisen et al. [1998], McLachlan et al. [2002]), and cancer classification (e.g., Golub et al. [1999], Alizadeh et al. [2000]).

Detection of co-expressed genes from microarray data has attracted much attention since many coexpressed genes are found to have functional relationships, e.g., lying in the same signal transduction pathway (Eisen et al., 1998; DeRisi et al., 1997). Hierarchical clustering (Eisen et al., 1998) and relevance network construction (Butte and Kohane, 2000; Farkas et al., 2003) are two important exploratory techniques. Both of these techniques are based on discovering pairs of co-expressed genes, which is one of the fundamental objectives in functional genomics and system biology. While not necessarily true in many higher eukaryotes (Boutanaev et al., 2002), pairwise gene co-expression as prescribed by the standard two-component model (Nixon et al., 1986) characterizes gene co-expression in bacteria, single-celled eukaryotes, archaea, and higher plants (Stock et al., 2000).

Clearly, there is a need for statistical methodology for high throughput screening of co-expressed gene pairs with stochastic error and strength of association controls. Two issues have to be considered in developing such a methodology, namely, choice of screening statistic and choice of screening acceptance and rejection criteria.

Regarding the choice of screening statistic, several methods have been adopted to measure the strength of association between expression profiles of gene pairs, such as the Pearson correlation coefficient (Zhou et al., 2002), coherence (Butte et al., 2001), mutual information (Butte and Kohane, 2000), edge detection (Filkov et al., 2002), and dominant spectral component analysis (Yeung et al., 2004). Each of these methods has advantages and disadvantages. The Pearson correlation coefficient has been one of the most popular choices because it is easily computed and its performance is often comparable to more complex and computational intense methods (Yeung et al., 2004). However, the Pearson correlation coefficient only can capture linear relationships between gene expression profiles. To circumvent this limitation, we propose to use the nonparametric Kendall rank correlation coefficient that is able to capture both linear and nonlinear associations between gene expression profiles. Both linear and nonlinear associations are very common in cellular gene expression profiles; for example, two functionally related enzymes with similar catalytic activities may have a linear correlation between their expression profiles, while the two enzymes with very different catalytic activities may have a nonlinear correlation. The Pearson and Kendall correlation coefficient measures are especially convenient because their asymptotic distributions are available, as required by our two-stage screening procedure to be described below.

Regarding the choice of screening acceptance criteria, one approach is to calculate a sample correlation for each pair of genes and then to select the top pairs by correlation thresholding (e.g., Zhou et al. [2002] and Farkas et al. [2003]), e.g., those exceeding a minimum acceptable strength (MAS) level specified by the threshold. Without a statistical inference procedure, the observed weakly correlated gene pairs are more likely to due to chance, noise, etc., and hence are less likely to be biologically relevant. For this reason, the approach controls biological significance. However, it does not account for statistical sampling uncertainty and thus does not control error rate. Another approach (Lee et al., 2004) is to control statistical significance in addition to biological significance. It is implemented as a two-stage procedure: screen co-expressed gene pairs whose strength of association is different from zero using $p$-value thresholding, e.g. as determined by a specified level of family-wise error rate (FWER) or false discovery rate (FDR), followed by a "hard" correlation thresholding. The approach is able to control error rate at correlation level zero but not at any nonzero correlation level.

The purpose of combining correlation thresholding with $p$-value thresholding is to control sampling error (e.g., Type I, II errors or false discoveries) and systematic error (e.g., nonfunctionally relevant correlations) incurred in the screening process. Indeed, the sampling error alone can be controlled by adopting a regular hypothesis testing scheme, and the systematic error alone can be controlled by a correlation thresholding. However, a reliable procedure for simultaneously controlling sampling error and systematic error has not been well developed.

In this paper, a new two-stage statistical hypothesis testing scheme is applied in order to decide on whether the strength of association is statistically significant at the specified positive MAS level. 
Stage I screens statistically significant co-expression gene pairs whose strength of correlation is different from zero. It is then followed by Stage II, in which a "soft" correlation thresholding (FDR confidence interval, FDR-CI) instead of a "hard" thresholding is applied. Our method is directly inspired by the twostage screen methodology of Hero et al. (2004) that controls both false discovery rate (FDR) and minimum acceptable difference (MAD) in detecting differentially expressed genes.

We demonstrate the application of our two-stage screening algorithm by constructing relevance networks and clustering co-expressed genes from yeast galactose metabolism data (Ideker et al., 2000). This data represents approximately 6,200 gene expression levels on two-color cDNA microarrays collected over 20 physiological/genetic conditions (nine mutant and one wild type strains incubated in either GAL-inducing or noninducing media). By applying our two-stage algorithm on this dataset, we achieved a high specificity $(83-100 \%)$ in discovering genes in the galactose metabolism pathway as described in Section 4.

The outline of the paper is as follows. In Section 3, we describe the proposed two-stage multicriteria approach. In Section 4, we first show that the approach indeed controls FDR at the specified MAS level using synthetic data and then illustrate it for yeast galactose metabolism data. In Section 5, we discuss advantages of our method, model assumptions, and restrictions.

\section{METHODS}

\subsection{Measures of the strength of association}

There are many possible discriminants for strength of association between two variables, which we generally denote as a real number $\Gamma$. Under a Gaussian linear hypothesis, the Pearson correlation coefficient $\rho$ is an appropriate metric. A robust distribution-free alternative is the Kendall rank correlation coefficient (Kendall's $\tau$ ). The Pearson (Bickel and Doksum, 2000) and Kendall (Hollander and Wolfe, 1999) correlation coefficients are special cases of the generalized correlation coefficient (Daniel, 1944). We define $\left\{g_{p}\right\}_{p=1}^{G}$ as the indices of $G$ gene probes on the microarray; $\left\{X_{g_{p}}\right\}_{p=1}^{G}$ as normalized probe responses (random variables); and $\left\{\left\{x_{g_{p(n)}}\right\}_{p=1}^{G}\right\}_{n=1}^{N}$ as realizations of $\left\{X_{g_{p}}\right\}_{p=1}^{G}$ under $N$ i.i.d. microarray experiments.

2.1.1. Pearson correlation coefficient. The population Pearson correlation coefficient between random variables $X_{g_{i}}$ and $X_{g_{j}}$ (defined as long as $\operatorname{var}\left(X_{g_{i}}\right)$, $\operatorname{var}\left(X_{g_{j}}\right)$ are positive) is

$$
\rho\left(X_{g_{i}}, X_{g_{j}}\right)=\frac{\operatorname{cov}\left(X_{g_{i}}, X_{g_{j}}\right)}{\sqrt{\operatorname{var}\left(X_{g_{i}}\right) \operatorname{var}\left(X_{g_{j}}\right)}} .
$$

The sample Pearson correlation coefficient $\hat{\rho}$ is an asymptotically consistent unbiased estimator of $\rho$ :

$$
\hat{\rho}_{i, j}=\frac{S_{X_{g_{i}}, X_{g_{j}}}}{\sqrt{S_{X_{g_{i}}, X_{g_{i}}} S_{X_{g_{j}}, X_{g_{j}}}}},
$$

where $S_{X_{g_{i}}, X_{g_{i}}}, S_{X_{g_{j}}, X_{g_{j}}}$, and $S_{X_{g_{i}}, X_{g_{j}}}$ are sample variances and covariances given by

$$
\begin{aligned}
& S_{X_{g_{i}}, X_{g_{i}}}=(N-1)^{-1} \sum_{n=1}^{N}\left(X_{g_{i}(n)}-\overline{X_{g_{i}}}\right)^{2}, \\
& S_{X_{g_{j}}, X_{g_{j}}}=(N-1)^{-1} \sum_{n=1}^{N}\left(X_{g_{j}(n)}-\overline{X_{g_{j}}}\right)^{2}, \\
& S_{X_{g_{i}}, X_{g_{j}}}=(N-1)^{-1} \sum_{n=1}^{N}\left(X_{g_{i}(n)}-\overline{X_{g_{i}}}\right)\left(X_{g_{j}(n)}-\overline{X_{g_{j}}}\right),
\end{aligned}
$$


and

$$
\overline{X_{g_{i}}}=N^{-1} \sum_{n=1}^{N} X_{g_{i}(n)}, \quad \overline{X_{g_{j}}}=N^{-1} \sum_{n=1}^{N} X_{g_{j}(n)}
$$

are sample means.

2.1.2. Kendall rank correlation coefficient. Kendall's $\tau$ statistic is a measure of correlation that captures both linear and nonlinear associations. The parameter $\tau$ is defined as $\tau=P_{+}-P_{-}$, where, for any two independent pairs of observations $\left(x_{g_{i(n)}}, x_{g_{j(n)}}\right),\left(x_{g_{i(m)}}, x_{g_{j(m)}}\right)$ from the population, $P_{+}=P\left[\left(x_{g_{i(n)}}-\right.\right.$ $\left.\left.x_{g_{i(m)}}\right)\left(x_{g_{j(n)}}-x_{g_{j(m)}}\right) \geq 0\right]$ and $P_{-}=P\left[\left(x_{g_{i(n)}}-x_{g_{i(m)}}\right)\left(x_{g_{j(n)}}-x_{g_{j(m)}}\right)<0\right]$. An unbiased estimator of $\tau$ is given by the Kendall $\tau$ statistic:

$$
\hat{\tau}_{i, j}=2 \sum \sum_{1 \leq n \leq m \leq N} \frac{K_{n m}}{N(N-1)}
$$

where $K_{n m}$ is a indicator variable defined as $K_{n m}=\operatorname{sgn}\left(x_{g_{i(n)}}-x_{g_{i(m)}}\right) \operatorname{sgn}\left(x_{g_{j(n)}}-x_{g_{j(m)}}\right)$ for each set of pairs drawn from $\left\{X_{g_{i}}\right\}_{i=1}^{G}$ and $\left\{X_{g_{j}}\right\}_{j=1}^{G}$.

\subsection{Hypothesis testing scheme}

To screen the strongly co-expressed pairs of $G$ genes on each microarray, we will simultaneously test the $\Lambda=\left(\begin{array}{c}G \\ 2\end{array}\right)$ pairs of composite hypotheses: $\left\{H_{\lambda}, K_{\lambda}: \lambda=\left(g_{i}, g_{j}\right)\right\}$.

$$
\begin{gathered}
H_{\lambda}: \Gamma_{g_{i}, g_{j}} \leq \text { cormin versus } K_{\lambda}: \Gamma_{g_{i}, g_{j}}>\text { cormin } \\
\quad \text { for } g_{i} \neq g_{j}, \text { and } g_{i}, g_{j} \in(1,2, \ldots G)
\end{gathered}
$$

Here cormin is the specified minimum acceptable strength of correlation. The sample correlation coefficient $\hat{\Gamma}_{i, j}\left(\hat{\rho}_{i, j}\right.$ or $\left.\hat{\tau}_{i, j}\right)$ could be thresholded to decide on pairwise dependency of two genes in the sample. When we must decide between the null hypothesis $H_{\lambda}$ and the alternative hypothesis $K_{\lambda}$ based on such a threshold test, there will generally be decision errors in the form of false positives (Type I errors: decide $K_{\lambda}$ when $H_{\lambda}$ is true) and false negatives (Type II errors: decide $H_{\lambda}$ when $K_{\lambda}$ is true). The per comparison error rate (PCER) is defined as the number of Type I errors over the number of independent trials, i.e. the probability of Type I error. The $p$-value is the probability that a more improbable sample could have been drawn from the population(s) being tested given the assumption that the null hypothesis is true.

For $N$ realizations of any pair of gene probe responses, $\left\{x_{g_{i(n)}}, x_{g_{j(n)}}\right\}_{n=1}^{N}$, we first calculate $\hat{\tau}_{i, j}$ or $\hat{\rho}_{i, j}$, respectively. For large $N$, the PCER $p$-values for $\rho_{i, j}$ or $\tau_{i, j}$ are

$$
\begin{aligned}
& p_{\rho_{i, j}}=2\left(1-\Phi\left(\frac{\tanh ^{-1}\left(\hat{\rho}_{i, j}\right)}{(N-3)^{-1 / 2}}\right)\right) \\
& p_{\tau_{i, j}}=2\left(1-\Phi\left(\frac{K}{N(N-1)(2 N+5) / 18^{1 / 2}}\right)\right)
\end{aligned}
$$

where $\Phi$ is the cumulative density function of a standard Gaussian random variable and $K=$ $\sum \sum_{1 \leq n \leq m \leq N} K_{n m}$. The above expressions are based on asymptotic Gaussian approximations to $\hat{\rho}_{i, j}$ (Bickel and Doksum, 2000) and to $\hat{\tau}_{i, j}$ (Hollander and Wolfe, 1999).

The PCER $p$-value refers to the probability of Type I error incurred in testing a single pair of hypotheses for a single pair of genes $g_{i}, g_{j}$. It is the probability that purely random effects would have caused $g_{i}, g_{j}$ to be erroneously selected based on observing correlation between this pair of genes only. When considering the $\Lambda$ multiple hypotheses for all possible pairs, two adjusted error rates have frequently been considered in microarray studies. These are family-wise error rate (FWER) and false discovery rate (FDR) (Benjamini and Hochberg, 1995). The FWER is the probability that the test of all $\Lambda$ pairs of hypotheses yields at least 
one false positive in the set of declared positive responses. In contrast, the FDR is the average proportion of false positives in the set of declared positive responses. The FDR is dominated by the FWER and is therefore a less stringent measure of significance. As in previous studies (Reiner et al., 2003), we adopt the FDR to control statistical significance of the selected gene pair correlations in our screening procedure.

\subsection{Two-stage screening procedure}

Select a level $\alpha$ of FDR and a level cormin of MAS significance levels. We use a modified version of the two-stage screening procedure proposed for gene screening by (Hero et al., 2004). This procedure consists of two stages, summarized in Fig. 1.

Stage I. For each gene pair $\lambda=\left(g_{i}, g_{j}\right)$ in the set $\mathcal{G}$ of all $\Lambda=\left(\begin{array}{c}G \\ 2\end{array}\right)$ gene pairs, test the simple null hypothesis:

$$
H_{\lambda}: \Gamma_{g_{i}, g_{j}}=0 \text { versus } K_{\lambda}: \Gamma_{g_{i}, g_{j}} \neq 0 \text {, for } g_{i} \neq g_{j} \text {, and } g_{i}, g_{j} \in(1,2, \ldots G)
$$

at FDR level $\alpha$. The step-down procedure of Benjamini and Hochberg (1995) is used to accomplish this.

Stage II. Suppose a number $\Lambda_{1}$ pairs of genes, denoted by the set $\mathcal{G}_{1} \subset \mathcal{G}$, pass the Stage I procedure. In Stage II, we first construct asymptotic PCER confidence intervals (PCER-CI's): $I^{\lambda}(\alpha)$ for each $\Gamma(\rho$ or $\tau)$ in subset $\mathcal{G}_{1}$. We convert these PCER-CI's into FDR confidence intervals (FDR-CI's): $I^{\lambda}(\alpha) \rightarrow I^{\lambda}\left(\Lambda_{1} \alpha / \Lambda\right)$ using the procedure of Benjamini and Yekutieli (2004). A gene pair in subset $\mathcal{G}_{1}$ is declared to be both statistically significant and biologically significant if its FDR-CI does not intersect the MAS interval [-cormin, cormin] (to be shown in Fig. 5). The set of all such gene pairs is called $\mathcal{G}_{2}$.

Stage I (step-down): control of FDR at MAS $=0$

1. Specify FDR level $\alpha$ and MAS level cormin.

2. Compute a list of PCER $p$-values: $p_{1}, p_{2}, \ldots, p_{\Lambda}$ corresponding to $\Lambda=\left(\begin{array}{c}G \\ 2\end{array}\right)$ pairs of composite hypotheses: $\left\{H_{\lambda}, K_{\lambda}: \lambda=\left(g_{i}, g_{j}\right)\right\}$ from $\left\{\hat{\boldsymbol{\rho}}_{i, j}\right\}$ or $\left\{\hat{\tau}_{i, j}\right\}$.

3. Sort the list of PCER $p$-values in increasing order, i.e. $p_{(1)}, p_{(2)}, \ldots, p_{(\Lambda)}$.

4. Find the index $k_{0}$ where $k_{0}=\max \left\{k: p_{(k)} \leq \frac{k \alpha}{\Lambda v}\right\}$.

5. Set initial screening $\mathcal{G}_{1}$ as those $k_{0}=\Lambda_{1}$ gene pairs having $p$-values: $p_{(1)}, p_{(2)}, \ldots, p_{\left(k_{0}\right)}$.

In step $4, v=1$ if the test statistics can be assumed statistically independent or positively dependent, where $v=\sum_{\lambda=1}^{\Lambda} \lambda^{-1}$ under the general dependency assumption.

Stage II: control of FDR and MAS = cormin.

1. Construct $\Lambda_{1}$ different $(1-\alpha) \times 100 \%$ PCER-CI's $I^{\lambda}(\alpha)$ for $\rho$ or $\tau$ of each gene pair in $\mathcal{G}_{1}$ (Appendix 5.1).

2. Convert these PCER-CI's into $\Lambda_{1}$ different $(1-\alpha) \times 100 \%$ FDR-CI's using formula (Benjamini and Yekutieli 2004): $I^{\lambda}(\alpha) \rightarrow I^{\lambda}\left(\Lambda_{1} \alpha / \Lambda\right)$.

3. Select the subset $\mathcal{G}_{2}$ containing $\Lambda_{2}$ of $\Lambda_{1}$ gene pairs whose FDR-CI's do not intersect [-cormin, cormin].

FIG. 1. Two-stage direct screening procedure yields a subset $\mathcal{G}_{2}$ of all possible gene pairs $\mathcal{G}$ whose strength of association exceeds MAS level cormin at FDR level $\alpha$. 
1. Compute a list of PCER $p$-values: $p_{1}, p_{2}, \ldots, p_{\Lambda}$ corresponding to $\Lambda=\left(\begin{array}{l}G \\ 2\end{array}\right)$ pairs of composite hypotheses: $\left\{H_{\lambda}, K_{\lambda}: \lambda=\left(g_{i}, g_{j}\right)\right\}$ from $\left\{\hat{\rho}_{i, j}\right\}$ or $\left\{\hat{\tau}_{i, j}\right\}$.

2. Sort the list of PCER $p$-values in increasing order, i.e. $p_{(1)}, p_{(2)}, \ldots, p_{(\Lambda)}$.

for any gene pair $\lambda_{0} \in\left\{g_{i}, g_{j}\right\}_{i, j=1}^{G}$ :

- Find the minimal $\alpha=\alpha\left(\lambda_{0}\right)$ such that the PCER-CI $I^{\lambda_{0}}(\alpha)$ does not intersect $[-$ cormin, cormin].

- Compute the integer index $\left.N\left(\alpha\left(\lambda_{0}\right)\right)=\sum_{k=1}^{\Lambda} I\left(p_{(k)}\right) k \leq \alpha\left(\lambda_{0}\right)\right)$, where $I(A)$ is an indicator function of the truth of statement A. The FDR $p$-value of the gene pair $\lambda_{0}$ is then simply $p_{i}$, where $i=N\left(\alpha\left(\lambda_{0}\right)\right)$.

endfor

FIG. 2. Inverse screening procedure allows the FDR $p$-value of a gene pair's $\left(\lambda_{0}\right)$ strength of association to be computed.

In many practical situations, the experimenter may not be comfortable in specifying a MAS or FDR criterion in advance. In this situation, it is useful to solve the inverse problem: what is the most stringent pair of criteria $\left(\alpha\right.$, cormin) that would cause a particular subset of gene pairs to be included in the screen $\mathcal{G}_{2}$. The inverse screening procedure is displayed in Fig. 2.

\section{RESULTS}

\subsection{Validating the two-stage algorithm}

3.1.1. Validating asymptotic null distribution. Here we verify that the proposed two-stage algorithm controls FDR at a specified MAS level using simulated data. Since the $p$-values are based on asymptotic distribution approximations (Equations (5) and (6)), we display in Fig. 3a the goodness of fit of the $\hat{\rho}$ sampling distribution to the Gaussian distribution using QQ plots. Note that there is good agreement to the Gaussian distribution for $N \geq 10$. Moreover, since the construction of confidence intervals requires estimation of sampling distribution variance, the accuracy of the variance approximation is vital. This can be evaluated by the mean squared approximation error $(M S E)$ for sample size $N$ :

$$
\begin{aligned}
M S E_{\rho}^{(N)} & =\Lambda^{-1} \sum_{1 \leq i<j \leq G}\left(S_{\tanh ^{-1}\left(\hat{\rho}_{i, j}\right)}^{(N)}-(N-3)^{-1 / 2}\right)^{2}, \\
M S E_{\tau}^{(N)} & =\Lambda^{-1} \sum_{1 \leq i<j \leq G}\left(S_{\hat{\tau}_{i, j}}^{(N)}-\left(\frac{2}{N(N-1)} \frac{2(N-2)}{N(N-1)^{2}} \sum_{i=1}^{N}\left(C_{r}-\bar{C}\right)+1-\hat{\tau}\right)\right)^{2},
\end{aligned}
$$

where $S_{\tanh ^{-1}\left(\hat{\rho}_{i, j}\right)}^{(N)}$ and $S_{\hat{\tau}_{i, j}}^{(N)}$ denote standard errors of $\tanh ^{-1}\left(\hat{\rho}_{i, j}\right)$ and $\hat{\tau}_{i, j}$ at the sample size $N$. The definitions of $C_{r}$ and $\bar{C}$ can be found in Appendix 5.1. The $\hat{\rho}$ variance approximations are seen to be in good agreement even for small sample sizes $(N>10)$ from Fig. $3 b$.

3.1.2. Validating the error control procedure. In order to validate our FDR and MAS error control procedure, we simulated pairwise gene expression data based on known population covariances (Appendix 5.2). The actual FDR at a MAS level is calculated as a ratio of the number of screened gene pairs whose corresponding population correlation parameters $\Gamma_{i, j}$ are less than the MAS level specified, divided by the total 

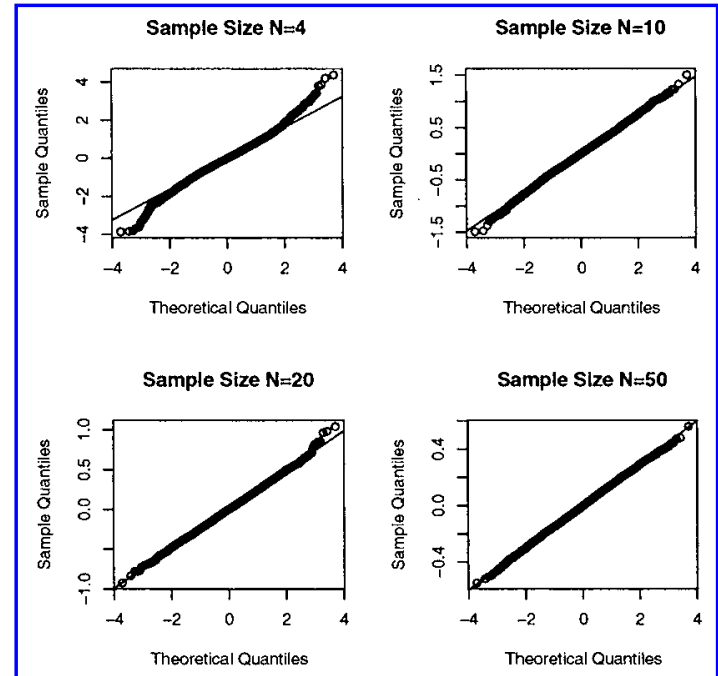

(a)

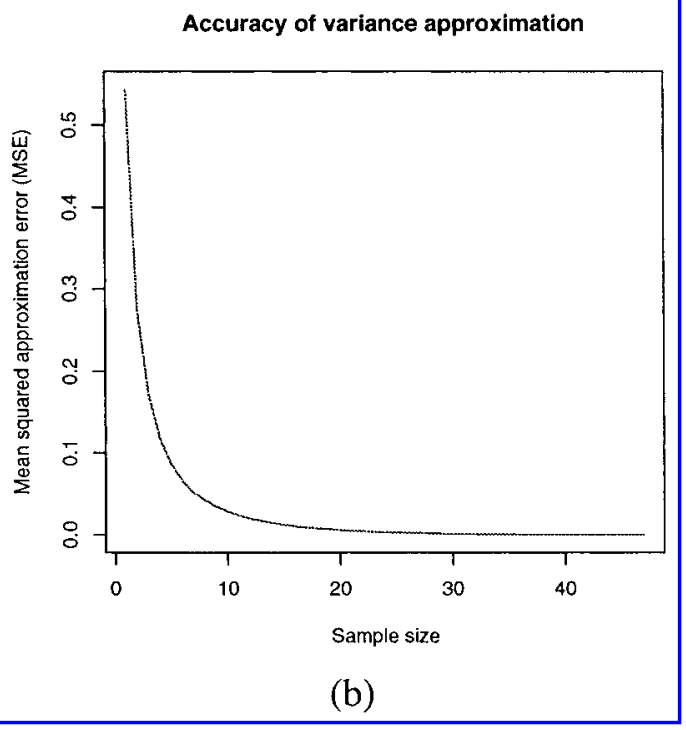

FIG. 3. Verification of Gaussian null sampling distribution and variance approximation for Pearson correlation coefficient (Equation (8)). (a) $Q Q$ plot of transformed sampling distribution of Pearson correlation coefficient $\hat{\rho}$ versus Gaussian distribution. (b) Mean squared approximation errors (MSE) of the variances of transformed sample Pearson correlation coefficients $\hat{\rho}$.

number of screened gene pairs. The actual MAS is the minimum true discovery of population correlation $\Gamma_{i, j}$ among the screened pairs. We specified 16 pairs of (FDR, MAS) criteria (four FDR levels: 0.2, 0.4, 0.6, 0.8; four MAS levels: 0.2, 0.4, 0.6, 0.8), and each is plotted as a different upper case English alphabet in Fig. 4. The 16 corresponding pairs of actual (FDR, MAS) criteria are also shown in Fig. 4 using the same set of lower case English alphabets. It can be observed that generally the actual FDR's (lower case) fall below the specified constraint (upper case) and the actual MAS's (lower case) fall above the specified constraints (upper case). Any deviations of actual FDRs and MASs from their specified levels are due to the conservative asymptotic approximation (Equations (5) and (6)). Observe that use of Kendall correlation (Fig. 4b) leads to greater overestimation of error rates than the Pearson correlation (Fig. 4a). Overestimation of error rates will translate into a reduction of power in discovering co-expressed pairs at the specified levels. 


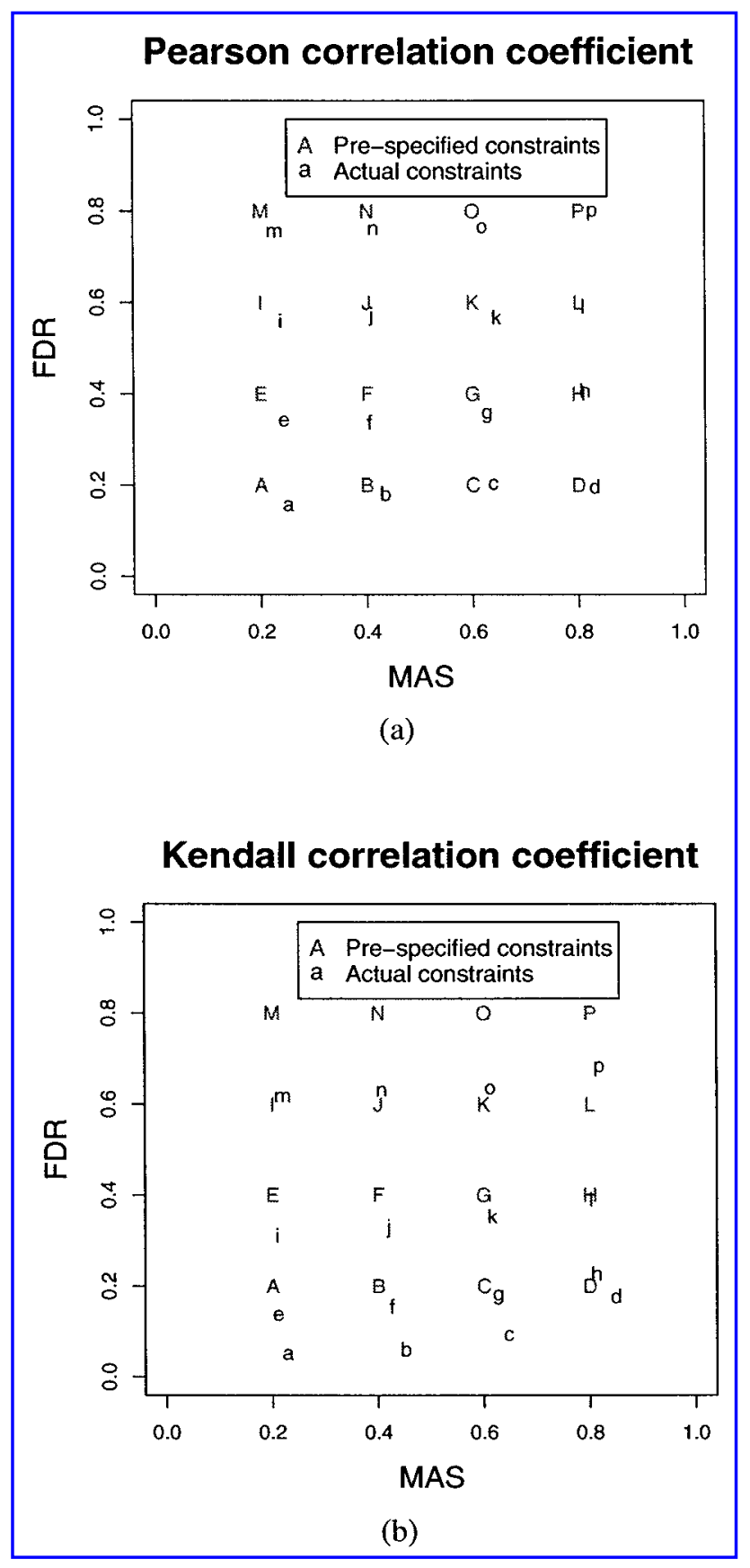

FIG. 4. Verification of two-stage error control procedure based on Pearson correlation coefficient (a) and Kendall correlation coefficient (b). Sample size $N=20$.

\subsection{Performance comparison}

We compared the performance of the two two-stage algorithms using the receiving operator characteristic (ROC) curve in which "sensitivity" is plotted against " 1 - specificity." Let $\Lambda_{0}$ denotes the number of false hypotheses (true strength of pairwise association is smaller than or equal to the threshold cormin), and let $\Lambda_{\alpha}$ denote the number of true hypotheses (true strength of pairwise association is greater than the threshold cormin). We counted false positives FP (falsely rejected hypotheses) and false negatives FN (falsely accepted hypotheses). The "sensitivity" (true positive rate, pTP) can be calculated as $p T P=$ $1-E\left(F N / \Lambda_{\alpha}\right)$; and the "1 - specificity" (false positive rate, $\left.\mathrm{pFP}\right)$ can be calculated as: $p F P=E\left(F P / \Lambda_{0}\right)$. 


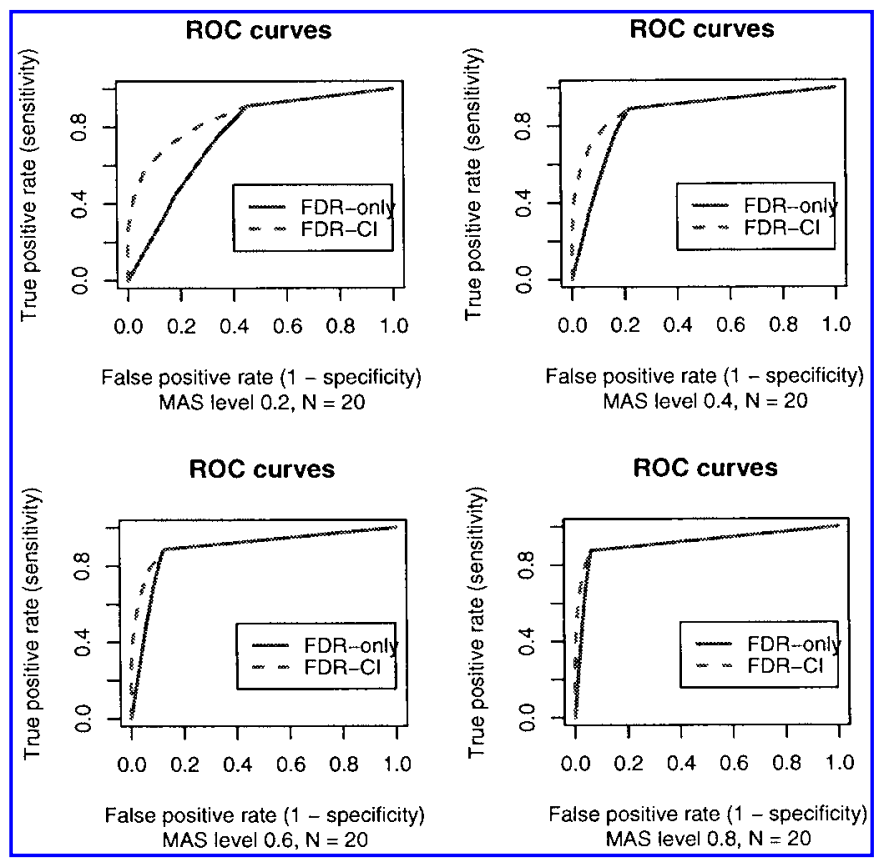

FIG. 5. ROC curves of "FDR-CI" test procedure and "FDR-only" test procedure based on Pearson correlation statistic.

The two-stage algorithm labeled as "FDR-only" in Fig. 5 denotes the FDR test followed by a "hard" correlation thresholding; and that labeled as "FDR-CI" denotes the FDR test followed by a "soft" correlation thresholding (FDR-CI). In Fig. 5, we observe overall better performance of FDR-CI test than the FDRonly test especially at low levels of correlation thresholding. For example, at the MAS level of 0.2 and the specificity level of 0.9 , the FDR-CI method has a three-fold higher sensitivity ( $p T P \approx 0.6$ ) than the FDR-only method ( $p T P \approx 0.2$ ).

\subsection{Constructing relevance networks with controlled FDR and MAS}

For the yeast galactose metabolism dataset, a subset of 997 differentially expressed genes were identified by Ideker et al. (2000) using a generalized likelihood ratio test procedure. Genes having a likelihood ratio statistic $\lambda \leq 45$ were selected as differentially expressed, i.e., whose mRNA levels differed significantly from the reference under one or more treatments.

Figures $6 \mathrm{a}$ and $6 \mathrm{~b}$ illustrate the direct implementation of the two-stage procedure to screen positively or negatively correlated gene pairs based on the Pearson correlation coefficient. The direct screening procedure is constrained by FDR level $\alpha=0.05$ and MAS level cormin $=0.5$. Stage I of the screen discovered $\Lambda_{1}=153,983$ out of $\Lambda=\left(\begin{array}{c}997 \\ 2\end{array}\right)=496,506$ gene pairs having FDR $\leq 0.05$, leaving 153,983 correlation coefficients for which FDR-CIs must be constructed. Recall that gene pair passes the Stage II screening if the FDR-CI does not intersect the interval $[-0.5,0.5]$. Of the 153,983 gene pairs, $\Lambda_{2}=18,135$ passed the Stage II screening and were declared to be both "biologically" and "statistically" significant. Similarly, using the Kendall correlation coefficient, there were $\Lambda_{1}=95,205$ gene pairs that passed the Stage I screen, and only $\Lambda_{2}=3,552$ gene pairs that passed the Stage II screen constrained by the same MAS and FDR criteria as above (see Table 1).

Although for Gaussian distributed pairs the Kendall rank correlation coefficient has lower discovery power compared to the Pearson correlation coefficient, our screening procedure was nevertheless able to pull out many nonlinearly correlated gene pairs that were missed by the Pearson correlation procedure. These nonlinearly correlated gene pairs, just like those linearly correlated ones, may be biologically relevant too. For example, the link between gene "RPC40" and gene "YDR516C" passed both Stage I and II screening $(\alpha=0.015$, cormin $=0.5)$ when using the Kendall correlation coefficient $(\hat{\tau}=-7.5 \mathrm{e}-01$, FDR $p$-value $=$ $6.2 \mathrm{e}-04$, FDR-CI $=[-9.7 \mathrm{e}-01,-5.4 \mathrm{e}-01])$, but they failed to pass even the first screening when the 


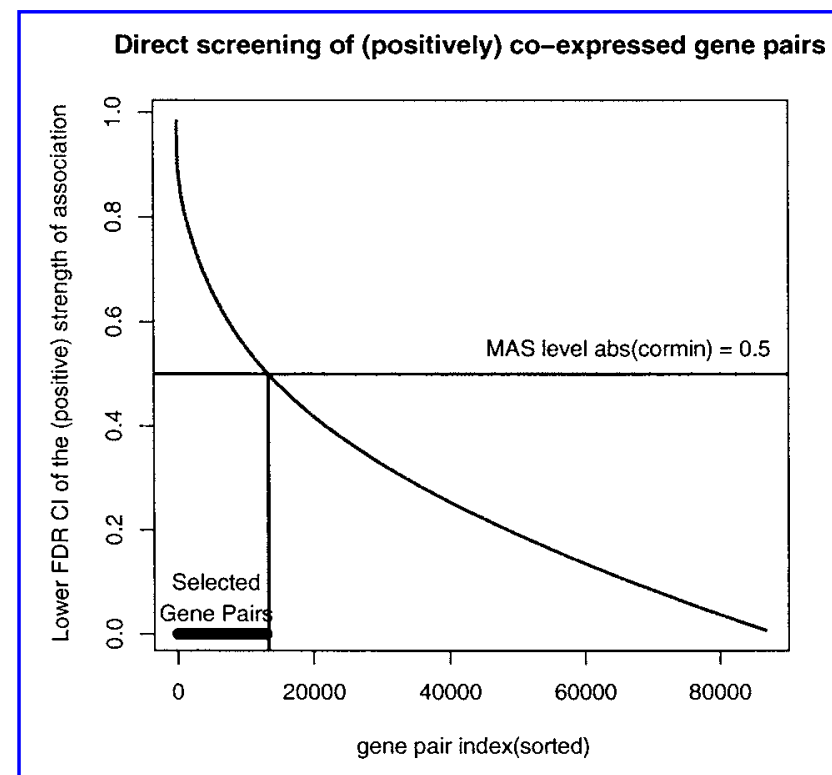

(a)

Direct screening of (negatively) co-expressed gene pairs

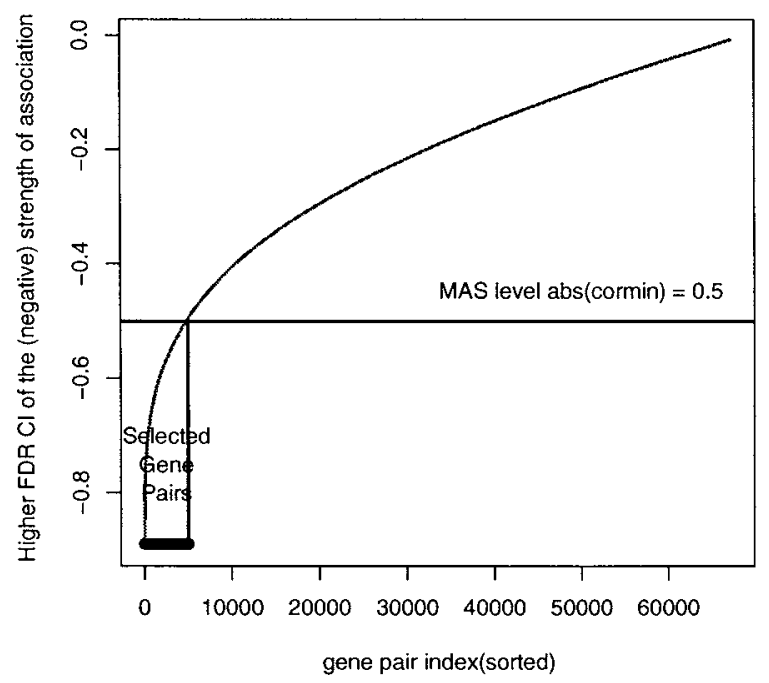

(b)

FIG. 6. Curves specify lower endpoints (a) and upper endpoints (b) of the 5\% FDR-CI's on the positive Pearson correlation coefficients (a) and negative Pearson correlation coefficients (b) for the galactose metabolism study. Only those gene pairs whose FDR-CI's do not intersect [-cormin, cormin] are selected by the second stage of screening. When the MAS strength of association criterion is cormin $=0.5$, these gene pairs are obtained by thresholding the curves as indicated.

Pearson correlation coefficient was used $(\hat{\rho}=-6.3 \mathrm{e}-01$, FDR $p$-value $=1.2 \mathrm{e}-02)$. From the scatter plot, we can observe an obvious nonlinear correlation for this gene pair (Fig. 7). The poor linear fit can be verified by fitting a simple linear regression model and observing $R^{2}=0.36$. Biologically, the gene RPC40 encodes RNA polymerase (I and III) subunit (transcription apparatus); although the specific function of gene "YDR516C" remains unclear, it was recently shown that it involves transcriptional induction of the 
Table 1. Top Ten "Hub Genes"a

\begin{tabular}{lclc}
\hline Gene name & Average rank & Gene name & Average rank \\
\hline RPL42B & 4.2 & RPS4A & 9.8 \\
RPS16B & 6.2 & RPL33A & 11.6 \\
RPL14A & 7.4 & RPL23B & 15.4 \\
RPS3 & 7.4 & RPS7A & 15.8 \\
GTT2 & 8.0 & RPS4B & 17.2 \\
\hline
\end{tabular}

${ }^{a}$ The rank of each gene is the average rank over five different networks. Each of five networks is constrained by a different pair of (FDR, MAS) criteria. The highest ranked gene is the most connected and stable gene under varying constraints of (FDR, MAS).

early meiotic-specific transcription factor IME1 (Dwight et al., 2002). Both genes are thus components of transcription apparatus. Applying our two-stage algorithm based on the Pearson correlation coefficient alone will miss the important functional relationship. Therefore, the Kendall correlation statistic can beat the Pearson correlation statistic in some instances, and hence the two correlation statistics should be used together to capture as many functional relationships as possible.

Relevance networks are implemented as a graph where $n$ nodes (genes) are connected by $p$ sets of edges (co-expressions). Each of the $p$ sets of edges are discovered using a different similarity measure (Butte et al., 2000; Butte and Kohane, 2000)). Therefore, our constructed networks are mixed networks with $p=2$ in which edges are discovered using either Pearson correlation coefficients or Kendall correlation coefficients constrained by the same set of (FDR, MAS) criteria. In relevance networks, genes that are of considerable interest to the biologist are "hub genes" such as RPL33A and RPS4A in Fig. 8. Hub genes are best connected genes that dominate a large part of the network topology (Jeong et al., 2001; Barabàsi, 2004). We constructed five such networks that are constrained by five pairs of constraints (FDR $\leq$ 0.05 , cormin $=0.5,0.6,0.7,0.8,0.9)$. Most of the "hub genes" in each discovered network fall into two categories: RPL and RPS. The former encodes Ribosome Protein Large (60S) subunit, and the latter encodes Ribosome Protein Small (40S) subunit. Both of these categories are structural components of the ribosome that is responsible for protein biosynthesis. Protein biosynthesis plays the central role in galactose metabolism because galactose is not a primary carbon source for yeast, when switching from primary carbon sources (glucose) to a secondary carbon source (e.g., galactose), many different types of proteins including transporters, enzymes, and regulators have to be synthesized to be able to degrade the secondary carbon source (Wieczorke et al., 1999). We ranked the hub genes by calculating and sorting average rank of each hub gene over five networks (Table 1). The list of hub genes are presumably indispensable for galactose metabolism (Jeong et al., 2001).

Figure 8 presents the discovered network topology with a FDR level of 5\% (5\% discovered edges are expected to be false positive) at the MAS level of cormin $=0.9$. The network is composed of 89 connected vertices and 132 edges. Similarly to some other biological networks, the network marginal degree distributions appear to be of the form of a power law. This was tested by verifying goodness of fit to the $\log$-transformed power-law model $\left(R^{2}=0.95\right)$, i.e., $\log P\left(D_{i}\right)=-\gamma \log D_{i}+\log \eta+\varepsilon_{i}$ (Barabàsi, 2004). Here, $\gamma$ and $\eta$ are shape and intercept parameters, $i$ is the index of a gene in the network, $\varepsilon_{i}$ is a residual fitting error, $D_{i}$ is the number of edges (degree) of $i$ th gene, and $P\left(D_{i}\right)$ is the corresponding probability.

\subsection{Clustering co-expressed genes}

Inspired by the Basic Local Alignment Search Tool (BLAST) (Altschul et al., 1990) and based on the "guilt-by-association" assumption (Eisen et al., 1998), we applied the two-stage screening procedure to cluster co-expressed genes with controlled FDR and MAS. We sought to demonstrate its application in metabolic pathway discovery by "rediscovering" the extensively studied galactose metabolic pathway, which consists of at least three types of genes including transporter genes (GAL2, HXTs, etc.), enzyme genes (GAL1, GAL7, GAL10, etc.) and transcription factor genes (GAL4, GAL80, GAL3, etc.). 


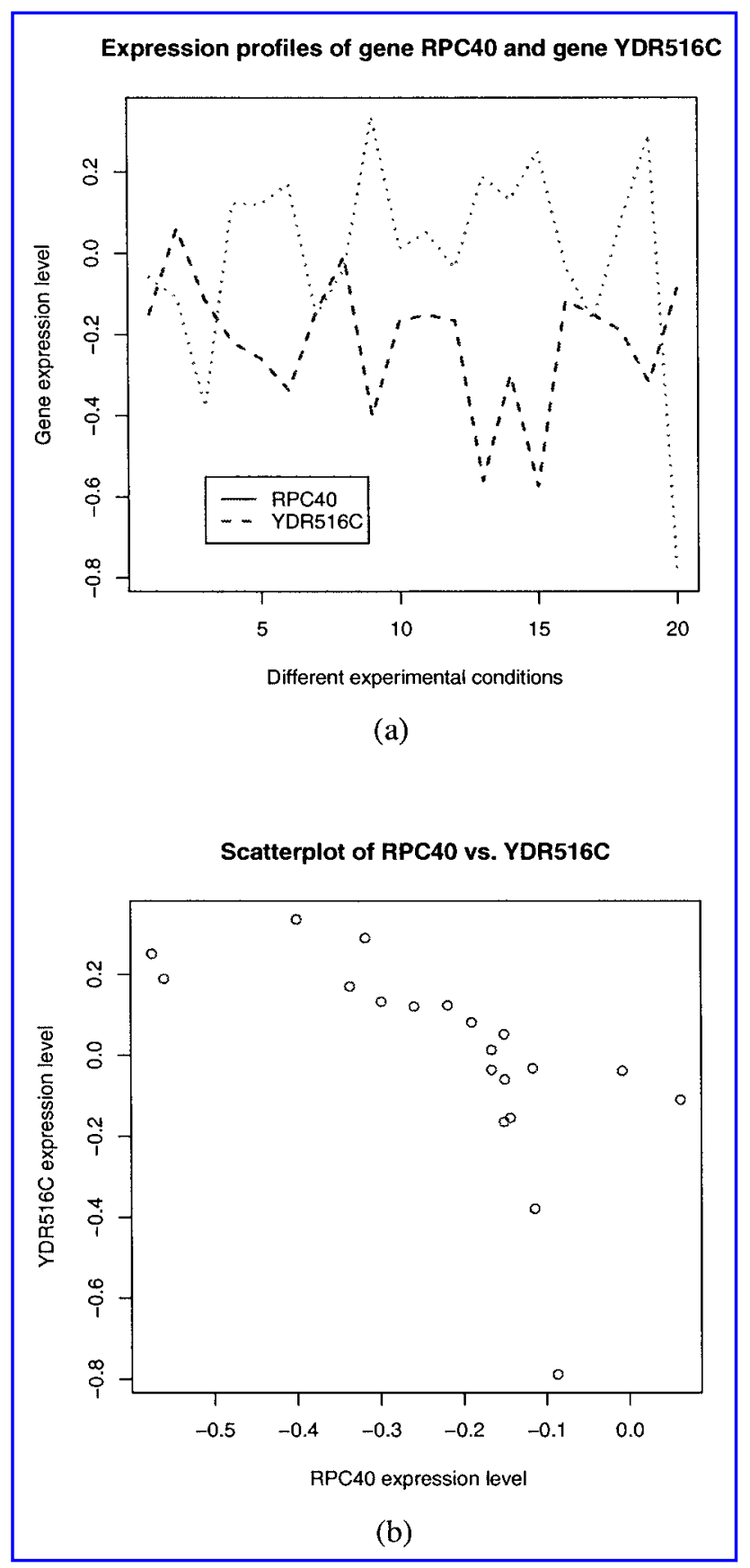

FIG. 7. A pair of nonlinearly correlated genes.

Some other genes are also involved in galactose metabolism but their roles are not entirely clear (Rohde et al., 2000; Ideker et al., 2001)). Therefore, our aims are not only to validate our procedure by rediscovering known co-expressed genes pairs, but also to discover some unknown genes in the pathway.

We selected gene GAL10 as the "seed gene" which encodes the UDP-glucose-4-epimerase (EC 5.1.3.3) (Fig. 9). We set a relatively stringent criterion $(\alpha=0.05$, cormin $=0.6)$, and cormin $=0.6$ is widely used in the literature (e.g., Zhou et al. [2002], Farkas et al. [2003]). We discovered six genes (GAL10, GAL7, GCY1, GAL1, GAL2, and YOR121C). Five of six genes are known to be lying in the pathway as shown in shaded squares in Fig. 9, which leads to a specificity of at least $83 \%$. The sixth gene YOR121C is a hypothetical ORF for which no functional annotation is currently available. Our results provide strong 


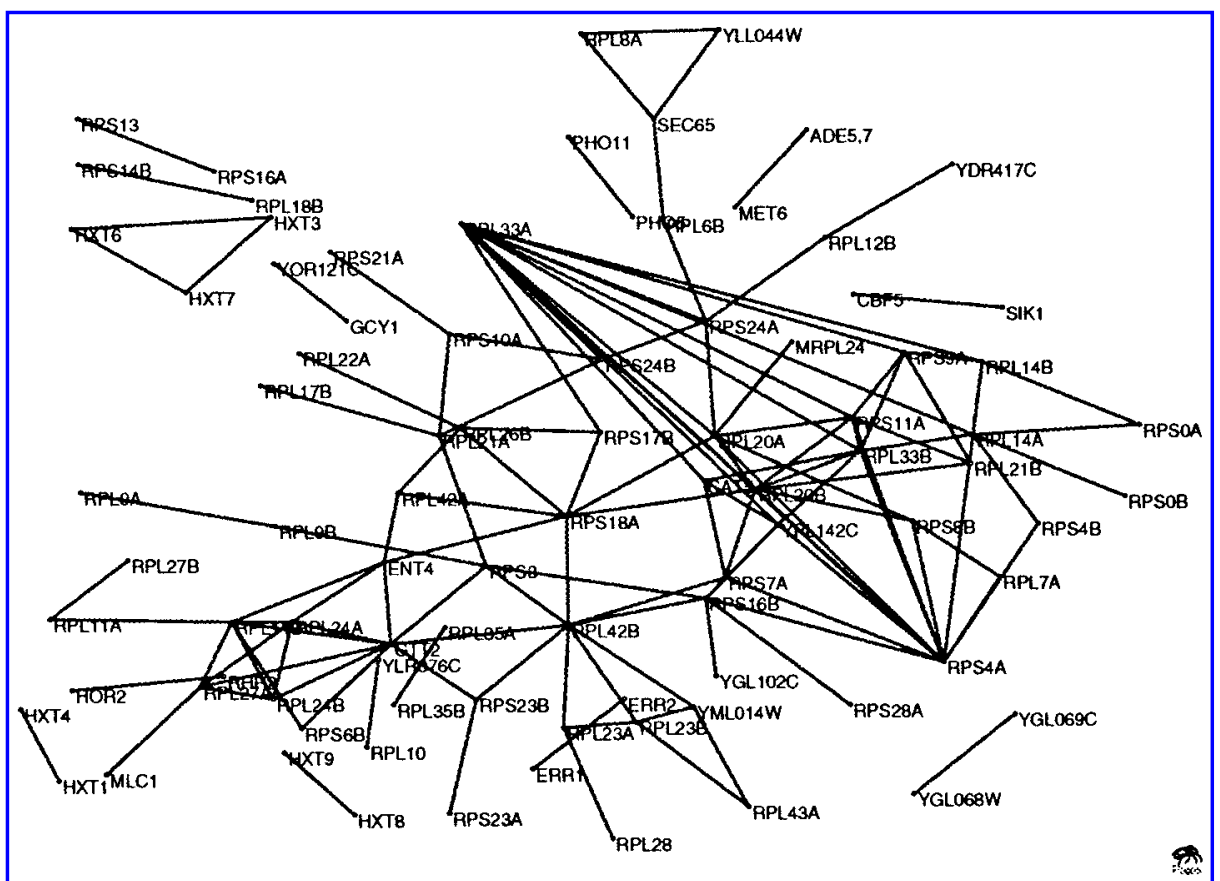

FIG. 8. Network topology visualization. The network is discovered by constraining FDR $\leq 5 \%$ at a MAS level of 0.9 . No significant negative correlation is discovered at this level. The graph is drawn using Pajek (Batagelj and Mrvar, 1998).

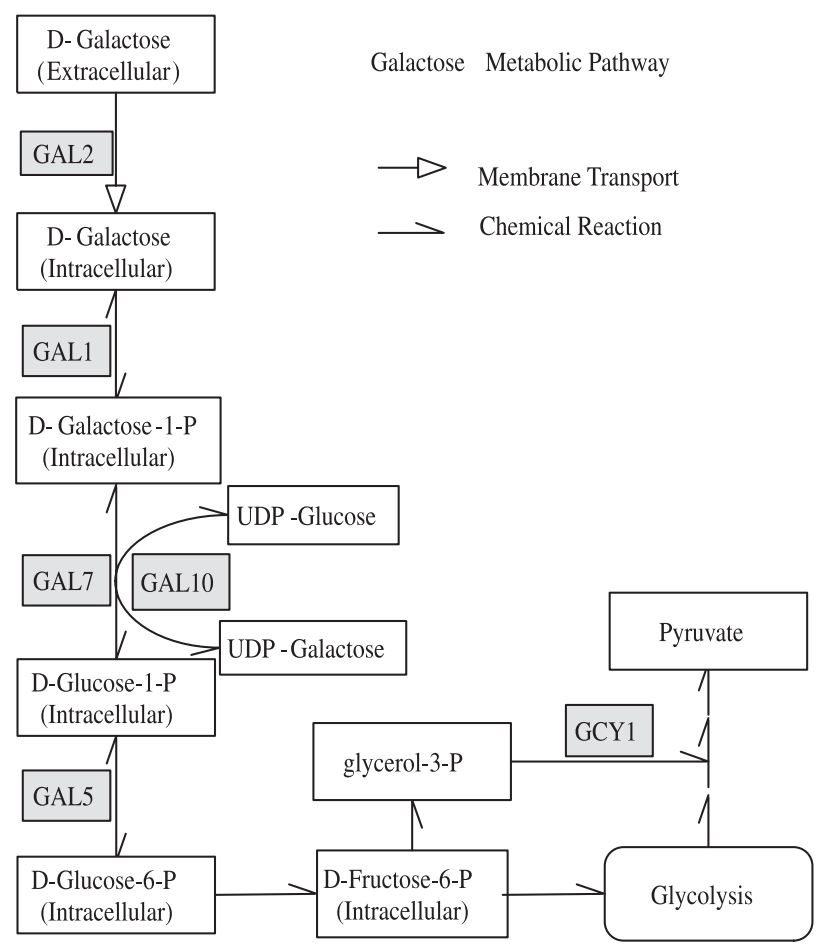

FIG. 9. Diagram of the structural module of the galactose metabolic pathway. The shaded boxes denote the five out of six genes whose gene products lie in the galactose metabolic pathway "rediscovered" by our algorithm. 
motivation to experimentally characterize this gene's biological function. Known transcription factor genes (GAL4 and GAL80) were not discoverable from this microarray experiment as the GAL4 and GAL80 expressions are time shifted and only one time sample was included. The pathways discovered using other seed genes in the pathway, such as GAL1 and GAL7, gave similar results.

\section{DISCUSSION}

In this paper, we presented a two-stage procedure for screening co-expressed gene pairs that controls both biological and statistical significance of the discovered strength of association. For the discovered co-expressions, our method also provides an "accuracy" assessment of the strength of association by constructing confidence intervals for the strength of each edge. Indeed, for the typically small sample size microarray data, a simultaneous confidence interval is useful to characterize reliability of the reported strength of association. Correlation thresholding is becoming standard practice in gene co-expression analyses (e.g., Butte and Kohane [2000], Butte et al. [2000], Zhou et al. [2002], Farkas et al. [2003], Lee et al.) [2004]), yet "hard" thresholding lowers the discriminative power of the FDR-based test (Fig. 5). Our "soft thresholding" procedure is able to control error rate and maintain discriminative power (Fig. 4). The method requires a tight confidence interval on correlation, which may be difficult to obtain for small sample sizes. However, we have shown that our algorithm provides error rate control at a biologically relevant level with relatively few samples (20 samples for Fig. 3b, Fig. 4).

The algorithm is sufficiently general to be applied to many different correlation measures, e.g., Spearman's or Hotelling's dependency statistics. The algorithm can also be extended to different frameworks, such as Gaussian graphic models (GGM), in which partial correlation coefficients are used as the dependency measures (Whittaker, 1990). Different groups have developed approaches to infer GGM from small sample size microarray data (Wang et al., 2003; Schafer and Strimmer, 2004; Dobra et al., 2004). Schafer and Strimmer recently presented a procedure that is based on the bootstrap estimator of the partial correlation coefficient (Schafer and Strimmer, 2004). Most of the pairwise partial correlations discovered by their procedure are very close to zero. On one hand, these ultra-weak correlations screened by the FDR-based inference procedure are "true correlation" from a pure statistical point of view. On the other hand, the "true correlation" may be caused by a variety of factors other than functional relationship, such as positional and spatial artifacts of gene co-expression along chromosomes (Kluger et al., 2003). Thus it seems necessary to combine such statistical testing with a "soft" thresholding to achieve high sensitivity and specificity (Fig. 5). This paper has presented such a method to simultaneously minimize the discovered proportion of the functionally irrelevant "true correlations" and maximize that of functionally relevant ones. Our two-stage algorithm has been extended to the GGM framework, and implementations are included in our R package "GeneNT" (available from www.cran.org).

The scope of application of our statistical analysis is explicitly that of randomly sampled, complete observational data (Dobra et al., 2004). In this paper, we are not concerned with developing models of causal gene networks (Dobra et al., 2004). This would require a different experimentation and interventation approach to understand directional influences, rather than the simple observational random sampling paradigm adopted here (Dobra et al., 2004).

Finally, we note that the two-stage procedures can be applied under the independency/positive dependency or the general dependency assumptions (Benjamini and Hochberg, 1995; Benjamini and Yekutieli, 2001). The implementation of the general dependency procedure $\left(v=\sum_{\lambda=1}^{\Lambda} \lambda^{-1}\right)$ causes loss of discovery power. The assumption of independence may not be critical in the discovery of relevance networks since biological networks are typically very sparse (Yeung et al., 2002).

\section{APPENDIX}

\subsection{Construct PCER-CI for $\rho$}

Based on the fact that $z\left(z=\tanh ^{-1}(\hat{\rho})\right)$ is the monotonic function of $\hat{\rho}$, the asymptotic PCER $(1-\alpha) \times 100 \%$ Confidence Interval: $I^{\lambda}(\alpha)$ on each true Pearson correlation coefficient $\rho$ of the set $\mathcal{G}_{1}$ is $\tanh \left(z-\frac{z_{\alpha / 2}}{(N-3)^{1 / 2}}\right) \leq \rho \leq\left(z+\frac{z_{\alpha / 2}}{(N-3)^{1 / 2}}\right)$, where $P\left(N(0,1)>z_{\alpha / 2}\right)=\alpha / 2$. 


\subsection{Construct PCER-CI for $\tau$}

The asymptotic PCER $(1-\alpha) \times 100 \%$ Confidence Interval: $I^{\lambda}(\alpha)$ on each true Kendall correlation coefficient $\tau$ of the set $\mathcal{G}_{1}$ is constructed as follows:

- Compute $C_{r}=\sum_{\substack{t=1 \\ t \neq r}}^{N} Q\left(\left(X_{r}, Y_{r}\right),\left(X_{t}, Y_{t}\right)\right)$, for $r=1,2, \ldots, N$, where $Q((a, b),(c, d))$ is given by:

$$
Q((a, b),(c, d))= \begin{cases}1 & \text { if }(d-b)(c-a)>0 \\ 0 & \text { if }(d-b)(c-a)=0 \\ -1 & \text { if }(d-b)(c-a)<0\end{cases}
$$

- Let $\bar{C}=\frac{1}{N} \sum_{r=1}^{N} C_{r}$ and define $\hat{\sigma}_{\tau}=\frac{2}{N(N-1)} \frac{2(N-2)}{N(N-1)} \sum_{i=1}^{N}\left[\left(C_{r}-\bar{C}\right)^{2}+1-\hat{\tau}^{2}\right]$

- $I^{\lambda}(\alpha): \hat{\tau}-z_{\alpha / 2} \hat{\sigma}_{\tau} \leq \tau \leq \hat{\tau}+z_{\alpha / 2} \hat{\sigma}_{\tau}$.

\subsection{Simulation of pairwise vectors based on prespecified population covariances}

\subsubsection{Pearson correlation coefficient $\rho$.}

- Specify a covariance matrix $\mathbf{V}$ and a mean vector $\mu$.

- Form the Cholesky decomposition of $\mathbf{V}$; i.e., find the lower triangular matrix $L$ such that $\mathbf{V}=L L^{T}$.

- Simulate a vector $\mathbf{z}$ with independent $N(0,1)$ elements.

- A vector simulated from the required multivariate normal distribution is then given by $\mu+L \mathbf{z}$.

\subsubsection{Kendall's $\tau$.}

- Specify a value for $\tau$.

- Simulate an $N \times N$ indicator matrix $M$ given $\tau$ as follows:

$$
M[n, m]_{1 \leq n<m \leq N}= \begin{cases}1 & \text { if Bernulli }\left(\frac{1+\tau}{2}\right) \text { is TRUE } \\ -1 & \text { if Otherwise. }\end{cases}
$$

- Simulate i.i.d pairs $\left(X_{r}, Y_{r}\right)(r=1,2, \ldots, N)$ according to $M$ matrix and definition

$$
Q((a, b),(c, d))= \begin{cases}1 & \text { if }(d-b)(c-a)>0 \\ -1 & \text { if }(d-b)(c-a)<0\end{cases}
$$

No tied observations are generated. Alternatively, $\hat{\tau}$ can be directly calculated from the indicator matrix $M$ without generating the i.i.d pairs (Equation (3)).

\section{ACKNOWLEDGMENTS}

This work was partially supported by grants from the National Institute of Health (EY01115), The Foundation Fighting Blindness, Sramek Foundation, and Research to Prevent Blindness.

\section{REFERENCES}

Alizadeh, A.A., Eisen, M.B., Davis, R.E., Ma, C., Lossos, I.S., Rosenwald, A., Boldrick, J.C., Sabet, H., Tran, T., Yu, X., Powell, J.I., Yang, L., Marti, G.E., Moore, T., Hudson, J. Jr., Lu, L., Lewis, D.B., Tibshirani, R., Sherlock, G., Chan, W.C., Greiner, T.C., Weisenburger, D.D., Armitage, J.O., Warnke, R., Levy, R., Wilson, W., Grever, M.R., Byrd, J.C., Botstein, D., Brown, P.O., and Staudt, L.M. 2000. Distinct types of diffuse large B-cell lymphoma identified by gene expression profiling. Nature 403, 503-511. 
Altschul, S., Gish, W., Miller, W., Myers, E.W., and Lipman, D.J. 1990. Basic local alignment search tool. J. Mol. Biol. 215, 403-410.

Barabàsi, A. 2004. Network biology: Understanding the cell's functional organization. Nat. Rev. Genet. 5, 101-113.

Batagelj, A., and Mrvar, A. 1998. Pajek-Program for large network analysis. Connections 21, 47-57.

Benjamini, Y., and Hochberg, Y. 1995. Controlling the false discovery rate-A practical and powerful approach to multiple testing. J. Roy. Stat. Soc. B Met. 57, 289-300.

Benjamini, Y., and Yekutieli, D. 2001. The control of the false discovery rate in multiple testing under dependency. Ann. Statist. 29, 1165-1188.

Benjamini, Y., and Yekutieli, D. 2004. False discovery rate adjusted multiple confidence intervals for selected parameters. J. Am. Statist. Assoc. 100, 71-80.

Boutanaev, A., Kalmykova, A., Shevelyov, Y.Y., and Nurminsky, D.I. 2002. Large clusters of co-expressed genes in the Drosophila genome. Nature 420, 666-669.

Bickel, P.J., and Doksum, K.A. 2000. Mathematical Statistics: Basic Ideas and Selected Topics, 2nd ed., Prentice Hall, Upper Saddle River, NJ.

Butte, A., Bao, L., Reis, B.Y., Watkins, T.W., and Kohane, I.S. 2001. Comparing the similarity of time-series gene expression using signal processing metrics. J. Biomed. Inf. 34, 396-405.

Butte, A., and Kohane, I.S. 2000. Mutual information relevance networks: Functional genomic clustering using pairwise entropy measurements. Pac. Symp. Biocomput. 5, 415-426.

Butte, A., Tamayo, P., Slonim, D., Golub, T.R., and Kohane, I.S. 2000. Discovering functional relationships between RNA expression and chemotherapeutic susceptibility using relevance networks. Proc. Natl. Acad. Sci. USA 97, 12182-12186.

Daniel, H. 1944. The relation between measures of correlation in the universe of sample permutations. Biometrika 33 , 129-135.

DeRisi, J., Iyer, V., and Brown, P.O. 1997. Exploring the metabolic and genetic control of gene expression on a genomic scale. Science 278, 680-686.

Dobra, A., Hans, C., Nevins, R., Yao, G., and West, M. 2004. Sparse graphical models for exploring gene expression data. J. Multivariate Anal. 90, 196-212.

Dwight, S.S., Harris, M.A., Dolinski, K., Ball, C.A., Binkley, G., Christie, K.R., Fisk, D.G., Issel-Tarver, L., Schroeder, M., Sherlock, G., Sethuraman, A., Weng, S., Botstein, D., and Cherry, J.M. 2002. Saccharomyces Genome Database (SGD) provides secondary gene annotation using the gene ontology (GO). Nucl. Acid Res. 30, 69-72.

Eisen, M., Spellman, P., Brown, P.O., and Botstein, D. 1998. Cluster analysis and display of genome-wide expression patterns. Proc. Natl. Acad. Sci. USA 95, 14863-14868.

Farkas, I., Jeong, H., Vicsek, T., Barabasi, A.L., and Oltvai, Z.N. 2003. The topology of transcription regulatory network in the yeast, Saccharomyces cerevisiae. Physica A 318, 601-612.

Filkov, V., Skiena, S., and Zhi, J. 2002. Methods for analysis of microarray time-series data. J. Comp. Biol. 9, 317-330.

Golub, T., Slonim, D., Tamayo, P., Huard, C., Gaasenbeek, M., Mesirov, J.P., Coller, H., Loh, M.L., Downing, J.R., Caligiuri, M.A., Bloomfield, C.D., and Lander, E.S. 1999. Molecular classification of cancer: Class discovery and class prediction by gene expression monitoring. Science 286, 531-537.

Hero, A.O., Fleury, G., Mears, A., and Swaroop, A. 2004. Multicriteria gene screening for analysis of differential expression with DNA microarrays. EURASIP J. Applied Signal Processing 1, 43-52.

Hollander, A., and Wolfe, D. 1999. Nonparametric Statistical Methods, Wiley-Interscience, Hoboken, NJ.

Ideker, T., Thorsson, V., Ranish, J.A., Christmas, R., Buhler, J., Eng, J.K., Bumgarner, R., Goodlett, D.R., Aebersold, R., and Hood, L. 2001. Integrated genomic and proteomic analyses of a systematically perturbed metabolic network. Science 292, 929-934.

Ideker, T., Thorsson, V., Siegel, A.F., and Hood, L.E. 2000. Testing for differentially expressed genes by maximumlikelihood analysis of microarray data. J. Comp. Biol. 7, 805-817.

Jeong, H., Mason, S., Barabasi, A.L., and Oltvai, Z.N. 2001. Lethality and centrality in protein networks. Nature 411, 41-42.

Kluger, Y., Yu, H., Qian, J., and Gerstein, M. 2003. Relationship between gene co-expression and probe localization on microarray slides. BMC Genomics $4,49$.

Lee, H., Hsu, A., Sajdak, J., Qin, J., and Pavlidis, P. 2004. Coexpression analysis of human genes across many microarray data sets. Genome Res. 14, 1085-1094.

Lockhart, D., Dong, H., Byrne, M.C., Follettie, M.T., Gallo, M.V., Chee, M.S., Mittmann, M., Wang, C., Kobayashi, M., Horton, H., and Brown, E.L. 1996. Expression monitoring by hybridization to high-density oligonucleotide arrays. Nature Biotechnol. 14, 1675-1680.

McLachlan, G., Bean, R., and Peel, D. 2002. A mixture model-based approach to the clustering of microarray expression data. Bioinformatics 18, 413-422.

Nixon, T., Ronson, C., and Ausubel, F.M. 1986. Two-component regulatory systems responsive to environmental stimuli share strongly conserved domains with the nitrogen assimilation regulatory genes ntrB and ntrC. Proc. Natl. Acad. Sci. USA 83, 7850-7854. 
Reiner, A., Yekutieli, D., and Benjamini, Y. 2003. Identifying differentially expressed genes using false discovery rate controlling procedures. Bioinformatics 19, 386-375.

Rohde, J., Trinh, J., and Sadowski, I. 2000. Multiple signals regulate GAL transcription in yeast. Mol. Cell. Biol. 20, 3880-3886.

Schena, M., Shalon, D., Davis, R.W., and Brown, P.O. 1995. Quantitative monitoring of gene expression patterns with a complementary DNA microarray. Science 270, 467-470.

Schafer, J., and Strimmer, K. 2004. An empirical Bayes approach to inferring large-scale gene association networks. Bioinformatics 1, 1-13.

Stock, M., Victoria, L., and Goudreau, P.N. 2000. Two-component signal transduction. Ann. Rev. Biochem. 69, $183-215$.

Tusher, V., Tibshirani, R., and Chu, G. 2001. Significance analysis of microarrays applied to the the ionizing radiation response. Proc. Natl. Acad. Sci. USA 98, 5116-5121.

Wang, J., Myklebost, O., and Hovig, E. 2003. MGraph: Graphical models for microarray data analysis. Bioinformatics $19,2210-2211$.

Wieczorke, R., Krampe, S., Weierstall, T., Freidel, K., Hollenberg, C.P., and Boles, E. 1999. Concurrent knock-out of at least 20 transporter genes is required to block uptake of hexoses in Saccharomyces cerevisiae. FEBS Lett. 464, 123-128.

Whittaker, J. 1990. Graphic Models in Applied Multivariate Statistics, Wiley, New York.

Yeung, L., Szeto, L., Liew, A.W., and Yan, H. 2004. Dominant spectral component analysis for transcriptional regulations using microarray time-series data. Bioinformatics 20, 742-749.

Yeung, M., Tegner, J., and Collins, J.J. 2002. Reverse engineering gene networks using singular value decomposition and robust regression. Proc. Natl. Acad. Sci. USA 99, 6163-6168.

Zareparsi, S., Hero, A.O., Zack, D.J., Williams, R., and Swaroop, A. 2004. Seeing the unseen: Microarray-based gene expression profiling in vision. Invest. Ophthalmol. Vis. Sci. 45, 2457-2462.

Zhou, X., Kao, M., and Wong, W.H. 2002. Transitive functional annotation by shortest path analysis of gene expression data. Proc. Natl. Acad. Sci. USA 99, 12783-12788.

Address correspondence to: Dongxiao Zhu Bioinformatics

2017H Palmer Commons University of Michigan Ann Arbor, MI 48109

E-mail: zhud@umich.edu 


\section{This article has been cited by:}

1. J. Hu, F. Hu. 2009. Estimating equation-based causality analysis with application to microarray time series data. Biostatistics 10:3, 468-480. [CrossRef]

2. Annely M. Richardson, Karen Woodson, Yonghong Wang, Jaime Rodriguez-Canales, Heidi S. Erickson, Michael A. Tangrea, Kristian Novakovic, Sergio Gonzalez, Alfredo Velasco, Ernest S. Kawasaki, Michael R. Emmert-Buck, Rodrigo F. Chuaqui, Audrey Player. 2008. Global Expression Analysis of Prostate Cancer-associated Stroma and Epithelia. Diagnostic Molecular Pathology 16:4, 189-197. [CrossRef]

3. Dongxiao Zhu , Alfred O. Hero III . 2007. Bayesian Hierarchical Model for Large-Scale Covariance Matrix EstimationBayesian Hierarchical Model for Large-Scale Covariance Matrix Estimation. Journal of Computational Biology 14:10, 1311-1326. [Abstract] [PDF] [PDF Plus]

4. Mark A. van de Wiel, Kyung In Kim. 2007. Estimating the False Discovery Rate Using Nonparametric Deconvolution. Biometrics 63:3, 806-815. [CrossRef] 